\title{
Hypoxia induces CXCR4 expression and biological activity in gastric cancer cells through activation of hypoxia-inducible factor-1 $\alpha$
}

\author{
YOON SUK OH, HA YON KIM, IK-CHAN SONG, HWAN-JUNG YUN, \\ DEOG-YEON JO, SAMYONG KIM and HYO JIN LEE \\ Department of Internal Medicine and Cancer Research Institute, Chungnam National University \\ School of Medicine, Daejeon 301-131, Republic of Korea \\ Received July 19, 2012; Accepted September 7, 2012
}

DOI: 10.3892/or.2012.2063

\begin{abstract}
Given the important role of CXCR4 in cancer metastasis, microenvironmental factors that modulate CXCR4 may have an impact on the process of tumor expansion. Hypoxia is a common feature of solid tumors and a significant microenvironmental factor that drives aggressive behavior. CXCR4 is upregulated in several cancer cells under hypoxic conditions, suggesting a relationship between tumor hypoxia and CXCR4. However, the role of hypoxia in regulating CXCR4 in gastric cancer remains poorly understood. KATO III gastric cancer cells were exposed to hypoxia or normoxia. CXCR4 expression in cells transfected with shRNA specific for HIF-1 $\alpha$ was investigated by western blotting and flow cytometry. Wound healing, migration and invasion assays were used to assess cell motility and the chemotactic response to CXCL12, a major CXCR4 ligand. CXCR4 expression at the protein level and in the cell membrane was significantly increased in KATO III cells following exposure to hypoxia. This upregulation of CXCR4 was implicated in increased cell motility and enhanced chemotactic responses (migration and invasion) to CXCL12 treatment in vitro. The increases in CXCR4 expression and metastatic potential in gastric cancer cells exposed to hypoxia were blocked by HIF-1 $\alpha$-specific shRNA. Our results indicate that hypoxia upregulates CXCR4 in gastric cancer cells in a HIF-1 $\alpha$-dependent manner, and that upregulation of CXCR4 plays a role in cancer cell migration and invasion. Thus, disrupting the hypoxia-HIF-1 $\alpha-\mathrm{CXCR} 4$ axis could be an attractive therapeutic strategy for the treatment of gastric cancer.
\end{abstract}

Correspondence to: Dr Hyo Jin Lee, Department of Internal Medicine and Cancer Research Institute, Chungnam National University School of Medicine, 6 Munwha-Dong, Jung-Gu, Daejeon 301-131, Republic of Korea

E-mail: cymed@cnu.ac.kr

Key words: gastric cancer, CXCR4, hypoxia, hypoxia-inducible factor- $1 \alpha$

\section{Introduction}

Gastric cancer is a major public health problem. Despite a decrease in incidence in developed countries, it is the fourth most common cancer worldwide and is the second leading cause of cancer-related mortality $(1,2)$. Although it is curable if detected early, most gastric cancers are asymptomatic until an advanced stage, when curative therapeutic approaches are not optimal (3). Therefore, to improve early diagnosis and develop new therapeutic strategies in gastric cancer, a better understanding of the molecular biology of gastric cancer development and progression is required. A variety of molecules, including chemokines and their receptors and growth factors, have been suggested to be responsible for the metastatic propensity of gastric cancer cells (3-5). However, the underlying molecular mechanisms that guide the directional migration and metastasis of cancer cells towards target tissues have yet to be established.

Chemokines and their receptors, particularly chemokine (C-X-C motif) ligand 12 (CXCL12) and its receptor chemokine (C-X-C motif) receptor 4 (CXCR4), have gained attention for their functional roles in the development and maintenance of the hematopoietic and immune systems, as well as in cancer metastasis and progression (4,6-8). CXCR4 has been demonstrated to be upregulated in a variety of different cancer cell lines and in human cancers including breast, prostate, ovarian, lung, and gastric cancer. It directs the migration, invasion and dissemination of tumor cells to specific sites that are rich in CXCL12, including lymph nodes, bone, the lungs and body cavities $(9,10)$. The CXCR4/CXCL12 axis is also involved in cancer cell proliferation at the primary or secondary site, or both, suggesting it might be the key determinant of overall disease progression. Indeed, in colorectal, breast, and gastric cancer, high expression of CXCR4 is associated with more aggressive tumor biology and poor survival outcome. CXCR4 is therefore considered a potential therapeutic target, and CXCR4 antagonists and other inhibitors have been shown to reduce tumor growth and metastasis in experimental models (9).

Given the critical role of CXCR4 in cancer development and progression, microenvironmental factors that modulate its 
levels may impact the process of tumor expansion. Intratumoral hypoxia is a common feature of solid tumors and an important microenvironmental factor that drives aggressive behavior in cancer $(9,11)$. After being exposed to hypoxia, several types of cancer cells increase their synthesis of a protein called hypoxia-inducible factor (HIF), which in turn binds to and transactivates target genes (12). HIF-1 is a heterodimeric transcription factor composed of a HIF-1 $\alpha$ subunit, which is rapidly degraded by ubiquitination and subsequent passage through the proteasomal pathway, a process that is inhibited under hypoxic conditions, and a HIF-1 $\beta$ subunit that is constitutively expressed. Accumulating evidence suggests that HIF-1 $\alpha$ plays a role in the regulation of CXCR4 expression and function. Moreover, the CXCR4 promoter contains four potential hypoxia-response elements upstream of the transcriptional start site, as well as one intra-intronic site (12-14). CXCR4 is upregulated on monocytes and endothelial cells, as well as tumor-associated macrophages and cancer cell lines, including melanoma and breast cancer cell lines, under hypoxic conditions (7,15-17). However, the role of hypoxia in regulating CXCR4 in gastric cancer remains poorly understood.

In the present study, we investigated the effect of hypoxia on CXCR4 expression and biological activity in gastric cancer cells and found that it promotes CXCR4 expression and facilitates tumor cell migration and invasion by a HIF-1 $\alpha$-dependent mechanism.

\section{Materials and methods}

Cell line and cultures. Human KATO III gastric cancer cell lines, purchased from the Korean Cell Line Bank (Seoul, Korea), were used in this study. Cells were cultured in RPMI-1640 medium (Gibco BRL Life Technologies, Grand Island, NY, USA) supplemented with $10 \%$ fetal bovine serum (FBS; Gibco BRL Life Technologies), $100 \mathrm{U} / \mathrm{ml}$ penicillin G, $100 \mu \mathrm{g} / \mathrm{ml}$ streptomycin (Sigma Aldrich, St. Louis, MO, USA) and $1 \mathrm{mmol} / 1$ L-glutamine (Gibco BRL Life Technologies). Cells were grown at $37^{\circ} \mathrm{C}$ in a humidified atmosphere containing $5 \% \mathrm{CO}_{2}$. For hypoxic exposure, cells were incubated with $5 \% \mathrm{CO}_{2}$ and $1 \% \mathrm{O}_{2}(\mathrm{v} / \mathrm{v})$, balanced with $\mathrm{N}_{2}$ gas, at $37^{\circ} \mathrm{C}$ for the indicated periods of time.

Cell-surface expression of CXCR4. After being cultured under either normoxic or hypoxic conditions for the indicated periods of time, cells were incubated with phycoerythrin (PE)-conjugated monoclonal anti-CXCR4 (12G5; BD Pharmingen, San Diego, CA, USA) at $4^{\circ} \mathrm{C}$ for $30 \mathrm{~min}$ and were analyzed using a Coulter Elite flow cytometer (Coulter Electronics Ltd., Hialeah, FL, USA). A PE-conjugated mouse IgG isotype-matched monoclonal antibody (dilution 1:50; Jackson ImmunoResearch Laboratories, Inc., West Grove, PA, USA) was used as a negative control.

Reagents. Human recombinant CXCL12/stromal cell derived factor-1 (SDF-1) was purchased from R\&D Systems, Inc. (Minneapolis, MN, USA), and cobalt chloride $\left(\mathrm{CoCl}_{2}\right)$ and AMD3100 were purchased from Sigma-Aldrich.

shRNA targeting HIF-1 $\alpha$ mRNA in gastric cancer cells. Knockdown of HIF-1 $\alpha$ in gastric cancer cells was achieved through lentivirus-mediated transduction of HIF-1 $\alpha$ mRNA-specific shRNA using Mission RNAi system clones (Sigma-Aldrich). To generate stable transfectants, lentiviral vectors and packaging vectors were cotransfected into HEK293T cells using Lipofectamine (Invitrogen, Carlsbad, CA, USA), according to the manufacturer's instructions. The following day, virus harvested from the supernatant was added to KATO III cells together with $10 \mu \mathrm{g} / \mathrm{ml}$ polybrene. After $24 \mathrm{~h}$, the medium was removed and replaced with fresh medium containing $8 \mu \mathrm{g} / \mathrm{ml}$ puromycin. Puromycin-resistant clones were selected after culture for 1 week in the presence of puromycin. HIF-1 $\alpha$ expression levels were analyzed by western blotting.

Wound healing, migration and invasion assays. Cells were grown to confluence in 6-well culture plates. A wound was created with a sterile pipette tip at an angle of $\sim 30^{\circ}$. After washing, the culture medium was replaced. Cell migration was monitored with a microscope for 24-48 h. For Transwell migration and invasion experiments, cells $\left(4 \times 10^{5}\right.$ per well) were loaded into the upper chamber of a 24-well Transwell plate containing an $8-\mu \mathrm{m}$ microporous membrane (CorningCostar, Cambridge, MA, USA) and were allowed to migrate into the lower chamber, which contained CXCL12 $(200 \mathrm{ng} / \mathrm{ml})$ or AMD3100 $(10 \mu \mathrm{M} / \mathrm{ml})$ or medium alone (RPMI-1640 supplemented with $10 \% \mathrm{FBS}$ ) at $37^{\circ} \mathrm{C}$ in normoxic or hypoxic conditions for $24 \mathrm{~h}$ (migration) or $48 \mathrm{~h}$ (invasion). For the migration assay, the lower surface of the filter was coated with $10 \mu \mathrm{g}$ of gelatin; for the invasion assay, the upper side was coated with $12 \mu \mathrm{g}$ of reconstituted basement membrane substance (Matrigel; BD Biosciences, Bedford, MA, USA). Cells were fixed and stained with hematoxylin and eosin. Non-migrating cells on the upper surface of the filter were removed by wiping with a cotton swab. Chemotaxis was quantified by counting the cells that migrated to the lower side of the filter under an optical microscope. Six random fields were counted for each assay.

Western blot analysis. Western blotting was used to detect protein molecules. Cells were starved in serum-free medium for $12 \mathrm{~h}$ and then stimulated with cytokines or CXCR4 antagonists. The cells were collected by centrifugation, washed in phosphate-buffered saline, and lysed by the addition of RIPA buffer (5 M NaCl, NP-40, sodium deoxycholate, $1 \mathrm{M}$ Tris- $\mathrm{HCl}$ (pH 7.4), 0.5 M EDTA (pH 8.0). Equal amounts of protein from each sample were separated by electrophoresis on $10 \%$ SDS-polyacrylamide gels and transferred to polyvinylidene fluoride membranes (Amersham Life Science, Arlington Heights, IL, USA). The membranes were blocked for $1 \mathrm{~h}$ in Tris-buffered saline (TBS) containing 5\% (w/v) milk and $0.1 \%$ Tween-20, and were then incubated overnight at $4^{\circ} \mathrm{C}$ with primary mouse or rabbit monoclonal antibody (Cell Signaling Technology Inc., Danvers, MA, USA). The blots were washed with TBS containing Tween-20, incubated with anti-mouse or anti-rabbit secondary antibody for $2 \mathrm{~h}$, and developed using West-Zol Plus (iNtRON Biotechnology, Seoul, Korea). The following antibodies were used: anti-CXCR4 monoclonal antibody (12G5; Thermo Scientific, Rockford, IL, USA) and anti-HIF-1 $\alpha$ polyclonal antibody (BD Biosciences).

Statistical analysis. Results are expressed as the means \pm standard deviation (SD) of at least three experiments. Data were 
A

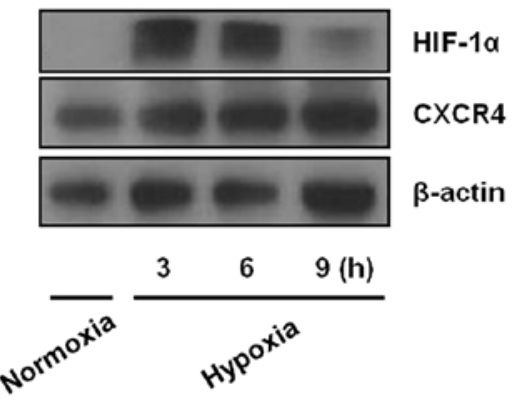

B

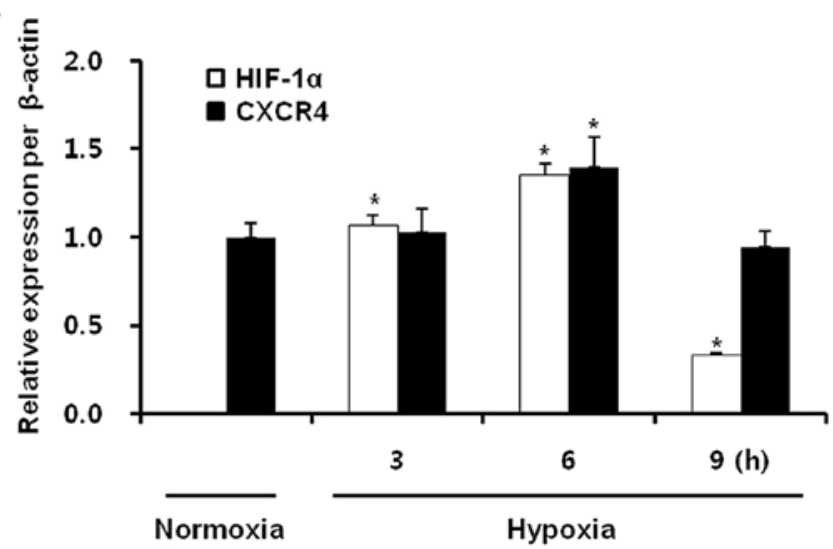

C
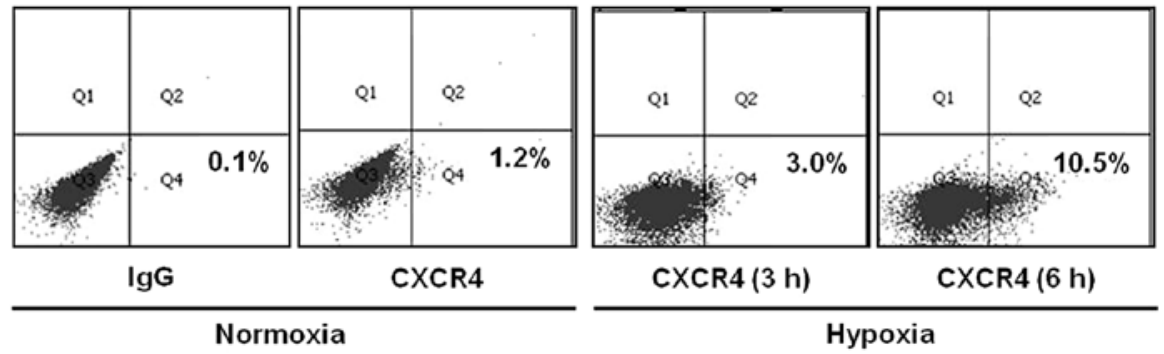

Figure 1. Hypoxia upregulates CXCR4 protein levels and its cell-surface expression. Cultured KATO III cells were grown under normoxic or hypoxic conditions for the indicated periods of time. (A and B) CXCR4 and HIF-1 $\alpha$ expression were upregulated by hypoxia, as determined by western blotting. Blots are representative of three independent experiments. Relative expression of CXCR4 and HIF-1 $\alpha$ protein was quantified by densitometry. Data are expressed as the means $\pm \mathrm{SD} ;{ }^{*} \mathrm{P}<0.05$ vs. normoxia. (C) Surface expression of CXCR4 in cells exposed to normoxia or hypoxia for the indicated periods of time was evaluated by flow cytometry using an antibody specific for CXCR4 (12G5). Representative results are shown.

analyzed using Student's t-test (for paired samples). $\mathrm{P}<0.05$ was considered to indicate statistically significant differences.

\section{Results}

Hypoxia upregulates CXCR4 protein levels and cell surface expression. To investigate the effect of hypoxia on CXCR4 expression in gastric cancer cells, KATO III cells were exposed to either normoxia $\left(21 \% \mathrm{O}_{2}\right)$ or hypoxia $\left(1 \% \mathrm{O}_{2}\right)$ for 3,6 or $9 \mathrm{~h}$ and the expression of HIF- $1 \alpha$ and CXCR4 proteins was analyzed by western blotting. Hypoxia upregulated CXCR4 and HIF-1 $\alpha$ protein levels. CXCR4 protein levels were increased at $6 \mathrm{~h}$ and then showed a decrease that corresponded to a change in HIF-1 $\alpha$ protein levels (Fig. 1A and B). Hypoxia also increased cell surface expression of CXCR4 (Fig. 1C). In addition, the hypoxia-mimetic agent $\mathrm{CoCl}_{2}$ increased CXCR4 protein levels and surface expression, responses that were accompanied by an increase in HIF-1 $\alpha$ levels (Fig. 2). These observations were consistent with the above hypoxic stimulation results. These data provide evidence that CXCR4 expression in gastric cancer cells is upregulated by hypoxia.

$H I F-1 \alpha$ is involved in the induction of CXCR4 expression by hypoxia. To further explore whether HIF-1 $\alpha$ was involved in the upregulation of CXCR4 expression by hypoxia, we generated KATO III cell clones with stable knockdown of HIF-1 $\alpha$. Specific depletion of HIF-1 $\alpha$ in these cells was accomplished by lentivirus-mediated transduction and expression of a HIF-1 $\alpha$ mRNA-specific shRNA. We effectively inhibited HIF-1 $\alpha$ expression using this system (Fig. 3A and C). When exposed to hypoxic conditions, cells transfected with a lentivirus containing scramble sequences showed upregulation of CXCR4 protein levels and surface expression, as expected (Fig. 3A and B). By contrast, hypoxia-induced CXCR4 upregulation under hypoxic conditions was blocked in cells transfected with shRNA directed against HIF-1 $\alpha$ (Fig. 3A and B). Similarly, $\mathrm{CoCl}_{2}$-induced upregulation of CXCR4 protein levels and surface expression was inhibited by abrogation of HIF-1 $\alpha$ (Fig. 3C and D). Collectively, these data suggest that the induction of CXCR 4 expression by hypoxia is mediated by HIF-1 $\alpha$.

Hypoxia promotes the migration and invasion of gastric cancer cells. To examine the effect of hypoxia on the metastatic ability of gastric cancer cells, we performed wound healing and Transwell migration and invasion assays. The ability to heal wounds was assessed in cells exposed to normoxia and hypoxia. Hypoxia was shown to facilitate wound healing in gastric cancer cells. However, the facilitation of wound healing by hypoxia was abrogated by knockdown of HIF-1 $\alpha$ (Fig. 4). When exposed to hypoxia for $24 \mathrm{~h}$, KATO III cells showed significantly increased migration in response to CXCL12 compared to cells exposed to normoxia. These effects were blocked by treatment with AMD3100 or knockdown of HIF-1 $\alpha$ (Fig. 5). Invasion analysis similarly showed that a hypoxia- 
A

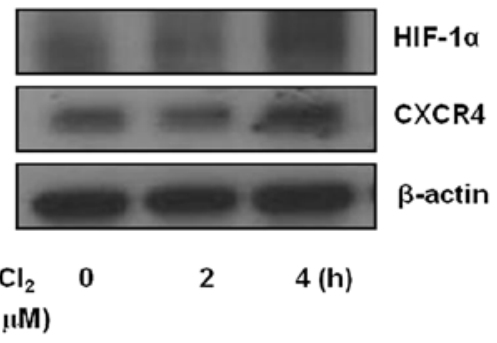

B

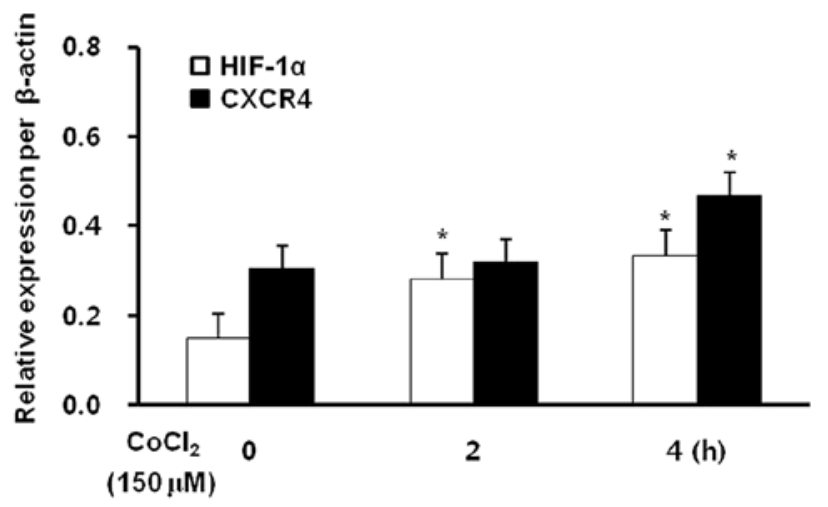

C

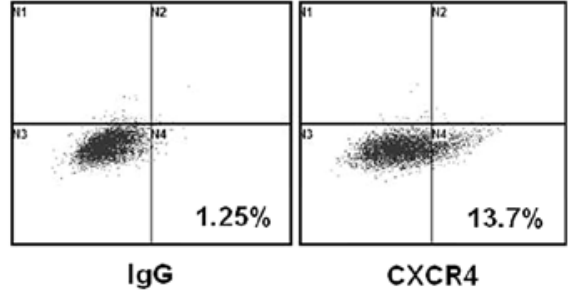

Normoxia

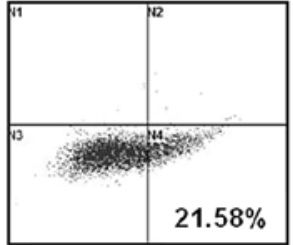

CXCR4 (4 h)

$\mathrm{CoCl}_{2}(150 \mu \mathrm{M})$

Figure 2. The hypoxia-mimetic agent $\mathrm{CoCl}_{2}$ increases CXCR4 protein levels and cell surface expression. Cultured KATO III cells were cultured with or without the hypoxia-inducing agent $\mathrm{CoCl}_{2}$ for the indicated periods of time. (A and B) CXCR4 and HIF-1 $\alpha$ expression were upregulated after treatment with CoCl${ }_{2}$, as determined by western blotting. Blots are representative of three independent experiments. Relative CXCR4 and HIF-1 $\alpha$ protein levels were quantified by densitometry. Data are expressed as the means $\pm \mathrm{SD} ;{ }^{*} \mathrm{P}<0.05$ vs. $0 \mathrm{~h}$. (C) Surface expression of CXCR4 was evaluated by flow cytometry using an antibody specific for CXCR4 (12G5) in cells treated with or without $\mathrm{CoCl}_{2}$ for the indicated periods of time. Representative results are shown.

A

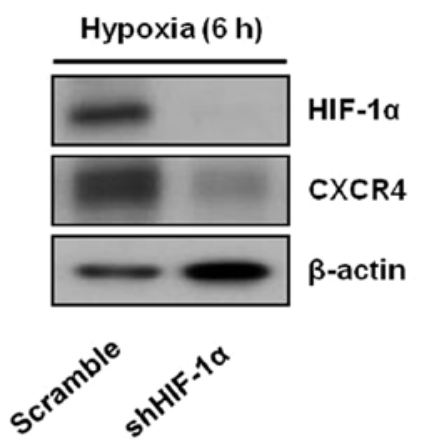

C

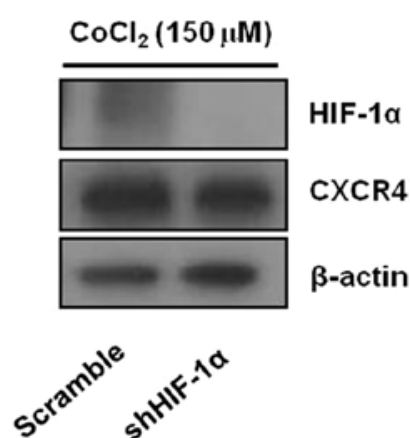

B

Hypoxia (6 h)

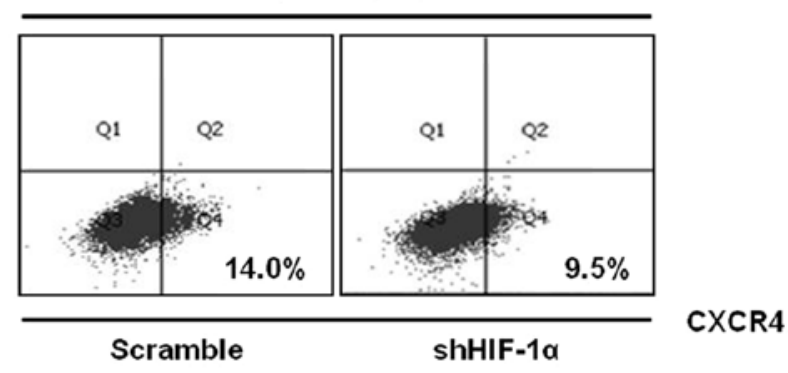

D $\mathrm{CoCl}_{2}(150 \mu \mathrm{M})$

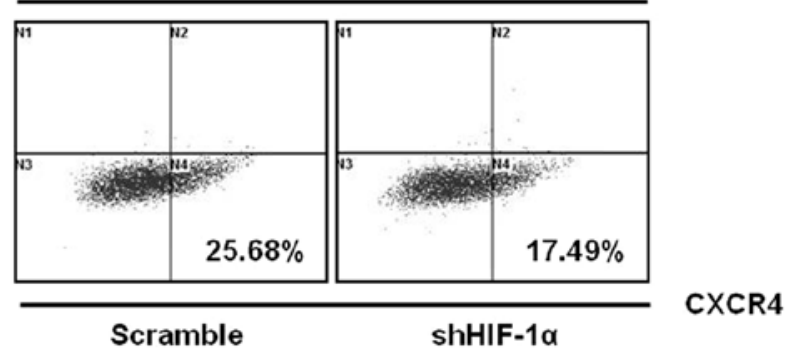

Figure 3. HIF-1 $\alpha$ is involved in the induction of CXCR4 expression by hypoxia. KATO III cells were stably transfected with HIF-1 $\alpha$ shRNA using a lentivirus, as described in Materials and methods. Three independent experiments were performed in triplicate; representative results are shown. (A) Stable transfectants were exposed to hypoxic conditions for $6 \mathrm{~h}$. The expression of HIF-1 $\alpha$ and CXCR4 was determined by western blotting. (B) Surface expression of CXCR4 in cells exposed to hypoxia for $6 \mathrm{~h}$ was evaluated by flow cytometry using an antibody specific for CXCR4 (12G5). (C) Stable transfectants were incubated with $\mathrm{CoCl}_{2}$ for $4 \mathrm{~h}$. The expression of HIF-1 $\alpha$ and CXCR4 was determined by western blotting. (D) Surface expression of CXCR4 in cells treated with CoCl ${ }_{2}$ for $4 \mathrm{~h}$ was analyzed by flow cytometry using an antibody specific for CXCR4. 


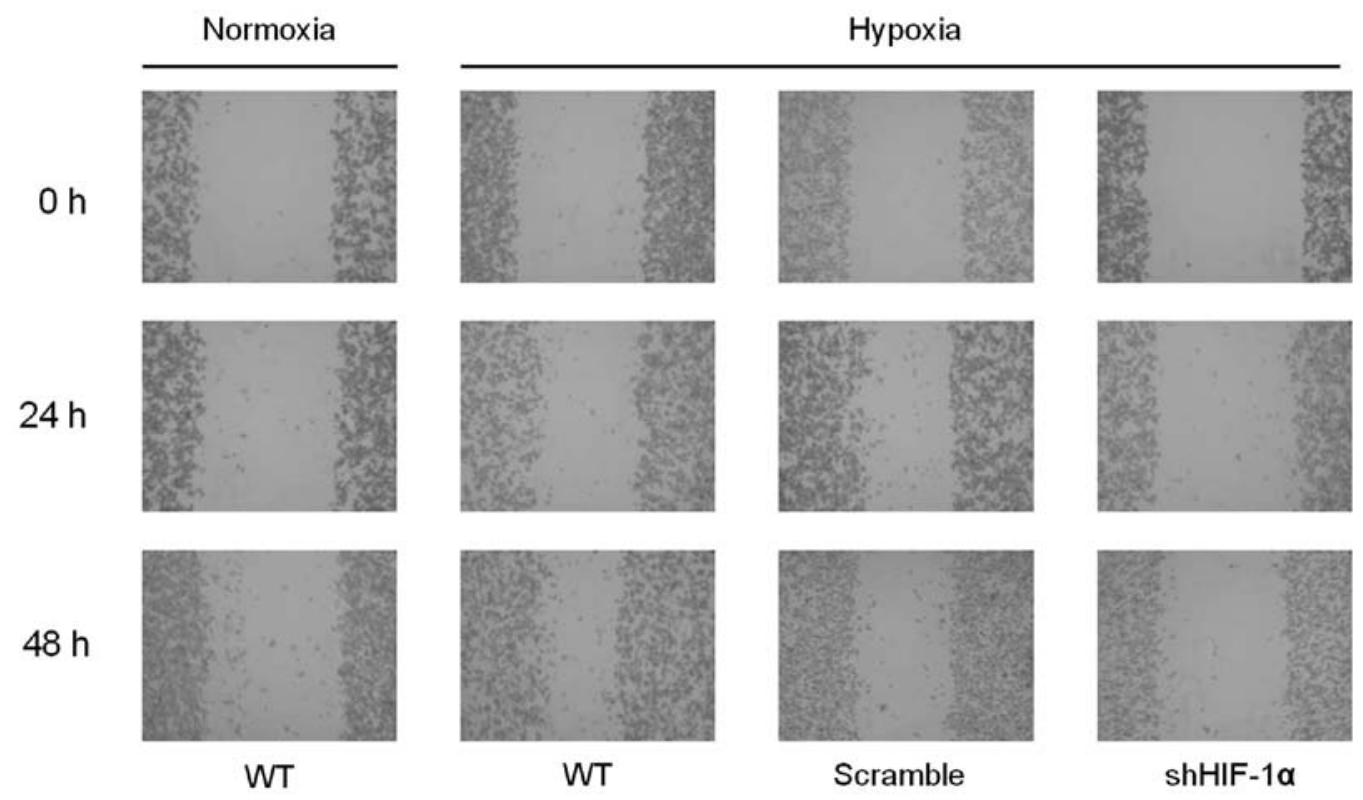

Figure 4. Hypoxia facilitates wound healing in gastric cancer cells. Confluent monolayers of KATO III cells were wounded and incubated for up to $48 \mathrm{~h}$ under normoxic or hypoxic conditions. Images were captured when the wounds were made (top) and after incubation for 24 (middle) and $48 \mathrm{~h}$ (bottom). WT, wild-type.

A

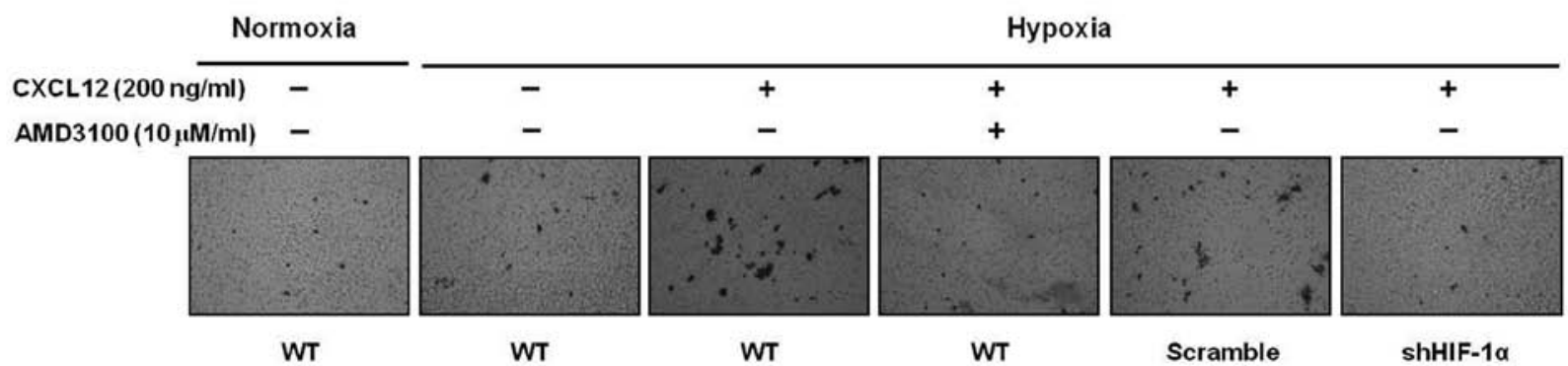

B

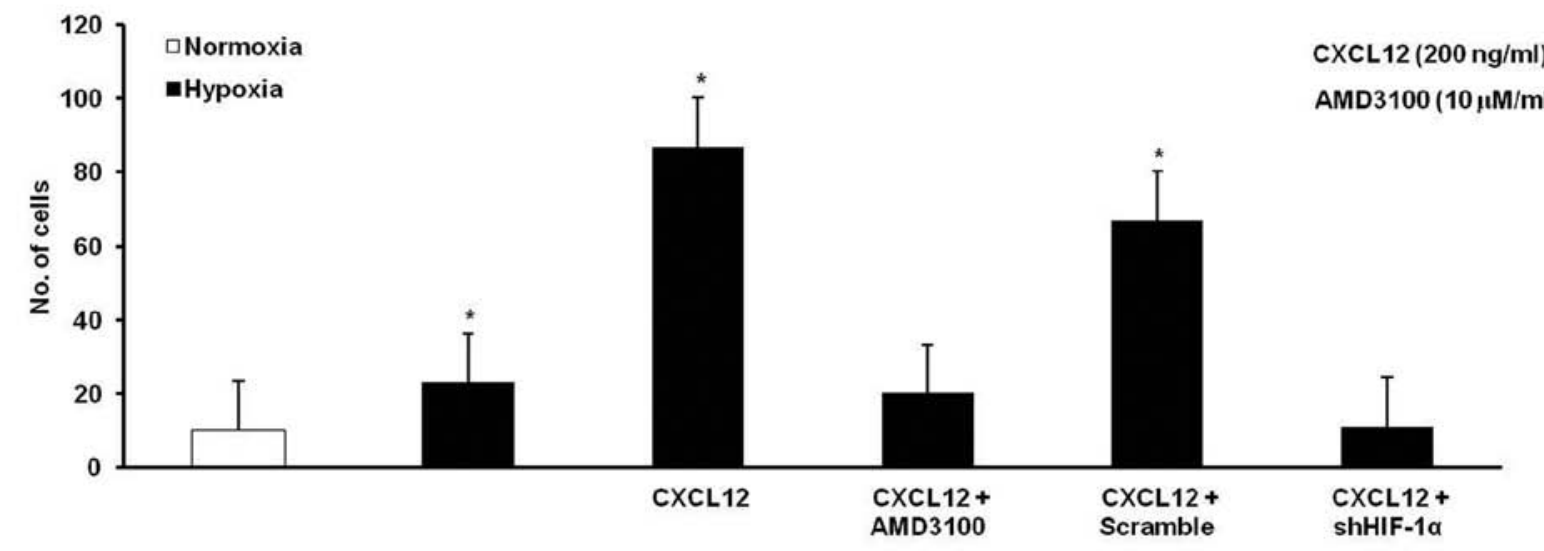

Figure 5. Hypoxia promotes gastric cancer cell migration. (A and B) KATO III cells were exposed to normoxia or hypoxia for $24 \mathrm{~h}$. Cell migration in response to CXCL12 was assessed as described in Materials and methods. Three independent experiments were performed in triplicate. Representative results are shown. Data are expressed as the means $\pm \mathrm{SD} ;{ }^{*} \mathrm{P}<0.05$ vs. normoxia. WT, wild-type.

induced increase in invasion in cells stimulated with CXCL12 was blocked by treatment with AMD3100 or knockdown of HIF-1 $\alpha$ (Fig. 6). These results suggest that hypoxia may induce gastric cancer cell migration and invasion in a HIF-1 $\alpha$ - and CXCR4-dependent manner.

\section{Discussion}

To the best of our knowledge, the present study is the first to provide evidence that hypoxia upregulates CXCR4 protein levels and cell membrane expression in gastric cancer cells, 
A

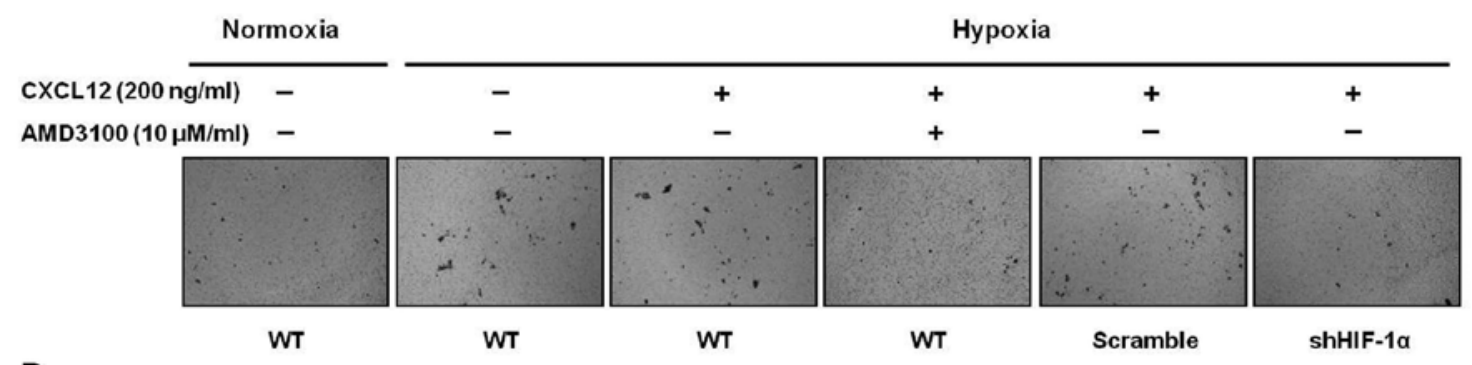

B

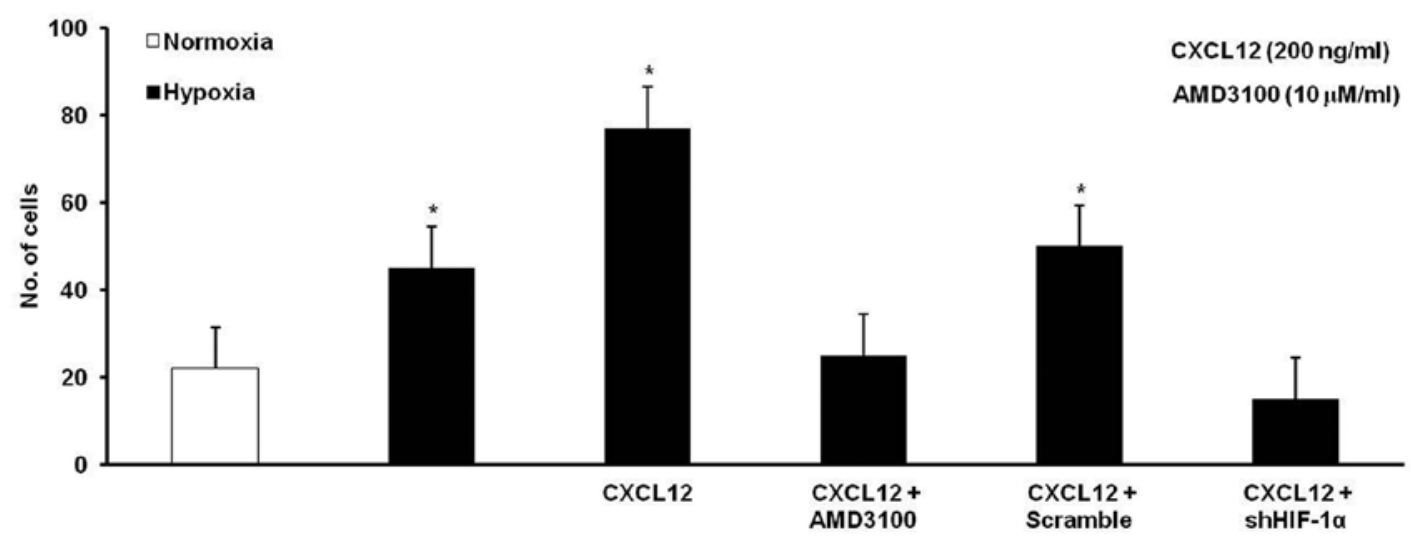

Figure 6. Hypoxia promotes gastric cancer cell invasion. (A and B) KATO III cells were exposed to normoxia or hypoxia for $48 \mathrm{~h}$. Cell invasion in response to CXCL12 was assessed as described in Materials and methods. Three independent experiments were performed in triplicate. Representative results are shown. Data are expressed as the means $\pm \mathrm{SD} ;{ }^{*} \mathrm{P}<0.05$ vs. normoxia. WT, wild-type.

responses that were dependent on activation of HIF-1 $\alpha$ and implicated in increased tumor cell migration and invasion.

Chemokines are 8- to 10-kDa chemoattractant cytokines that not only control leukocyte trafficking, but also play critical roles in the migration and metastasis of cancer cells (originating from primary tumors) with corresponding chemokine receptors to certain organs. Notably, the chemokine CXCL12 activates its receptor, CXCR4, which is involved in cancer cell migration and invasion, and thus promotes organ-specific localization of distant metastases from various carcinomas (18). In gastric cancer, accumulating evidence suggests that CXCR4 protein levels and cell membrane expression in vitro and in vivo are altered (4,19-22). The differential expression of CXCR4 in gastric cancer cells was also shown by gene expression profiling (23). Overexpression of CXCR4 in gastric cancer cells was associated with the development of peritoneal carcinomatosis, which is induced by dissemination of cancer cells into the peritoneal cavity $(20,24)$. Numerous studies have demonstrated that strong expression of CXCR4 is correlated with aggressive tumor characteristics such as deep invasion, lymph node, and liver metastasis (4,20,25-28). Furthermore, several pre-clinical investigations demonstrated that blocking of CXCR4 signaling reduced the size and number of tumor metastases $(20,29,30)$. Therefore, a better understanding of the mechanism by which CXCR4 expression and biological activity are regulated in gastric cancer is important for the development of improved treatment strategies.

In contrast to normal organs, oxygen homeostasis in solid tumors is deregulated and the $\mathrm{pO}_{2}$ decreases to very low levels as the tumors grow (15). Although hypoxia is cytotoxic to both normal and cancer cells, some cancer cells acquire characteristics that allow them to survive and grow under hypoxic conditions (such as, expression of angiogenic factors, glycolytic enzymes and stress proteins). In addition, these ischemic conditions contribute to the aggressive metastatic phenotypes of tumor cells, and metastasis, which is characterized by migration, seeding and growth of satellite lesions in specific organs, is commonly considered to be the final stage of cancer (11,31-34). Experimental and clinical data provided evidence for a relationship between intratumoral hypoxia and aggressive behavior in solid tumors, including gastric cancer (35-39). Several recent studies provide evidence that hypoxia, primarily acting through HIF- $1 \alpha$, is involved in the upregulation of CXCR4 in carcinomas and hematologic malignancies such as lung, breast cancer and myeloma, with HIF- $1 \alpha$ binding directly to the CXCR4 promoter (15,40-45). In this study, hypoxia increased CXCR4 protein levels and cell surface expression by activating HIF-1 $\alpha$, thereby supporting previous findings from other cancer cell lines and suggesting that tumor hypoxia plays a crucial role in the regulation of CXCR4 (15,16,41).

Next, we investigated whether hypoxia-induced upregulation of CXCR4 in gastric cancer cells was related to increased metastatic potential. Gastric cancer cells exposed to hypoxia showed significantly increased migration and invasion in response to CXCL12 treatment compared with those exposed to normoxia. The increase in migratory ability due to hypoxia was abrogated by knockdown of HIF- $1 \alpha$ and inhibition of CXCR4. These results are in accordance with previous reports in different types of cancer $(16,46)$ and suggest that 
upregulation of CXCR4 by HIF-1 $\alpha$ in response to hypoxia plays a biological role in gastric cancer cell migration. We previously reported that functional CXCR4 plays an important role in cancer cell migration during normoxia in gastric and gallbladder cancers $(18,21)$, suggesting that the CXCR4/ CXCL12 axis may be significantly involved in cancer progression and may be a potential target for therapeutics that block the interaction between CXCL12 and CXCR4, or that inhibit downstream signaling, in the treatment of cancer. To date, several antagonists have been used to target CXCR4, including AMD3100, T22 and ALX40-4C (10). Among them, AMD3100 (trade name Plerixafor) was shown to be well-tolerated and has been approved by the Food and Drug Administration of the United States for use in non-Hodgkin's lymphoma and multiple myeloma to mobilize stem cells for collection prior to autologous transplantation (47). AMD3100 is currently being tested against hematologic malignancies such as acute myeloid leukemia in early clinical trials in combination with chemotherapy with the rationale that disrupting the interaction between leukemic cells and the bone marrow microenvironment will increase the cytotoxic effect of chemotherapy (48).

In gastric cancer, several pre-clinical investigations demonstrated that monoclonal antibodies and specific low-molecular weight antagonists of CXCR4 showed antitumor activity in vitro and in vivo. CXCL12-induced cancer cell migration was significantly reduced by treatment with a neutralizing anti-CXCR4 antibody or AMD3100. AMD3100 reduced tumor growth and malignant ascites formation in a xenograft model $(20,21,29,49)$. Taken together, these findings suggest that hypoxia may increase CXCR4 activity in gastric cancer cells by activating HIF-1 $\alpha$, thereby resulting in increased migratory ability. This suggests that the hypoxia-HIF-1 $\alpha-\mathrm{CXCR} 4$ axis might be a potential therapeutic target.

In conclusion, hypoxia upregulates CXCR4 protein levels and cell membrane expression in gastric cancer cells in a HIF-1 $\alpha$-dependent manner. The upregulation of CXCR4 plays roles in cancer cell migration and invasion, which were reduced by knockdown of HIF-1 $\alpha$ or inhibition of CXCR4. Our results collectively suggest that disruption of the hypoxiaHIF-1 $\alpha$-CXCR4 axis is a potential therapeutic strategy for the treatment of gastric cancer.

\section{Acknowledgements}

This study was supported in part by the Basic Science Research Program through the National Research Foundation of Korea (NRF) funded by the Ministry of Education, Science and Technology (KRF-2009-0076540, KRF-2009-0067256).

\section{References}

1. Kamangar F, Dores GM and Anderson WF: Patterns of cancer incidence, mortality, and prevalence across five continents: defining priorities to reduce cancer disparities in different geographic regions of the world. J Clin Oncol 24: 2137-2150, 2006.

2. Lee HJ, Cho do Y, Park JC, et al: Phase II trial of biweekly paclitaxel plus infusional 5-fluorouracil and leucovorin in patients with advanced or recurrent inoperable gastric cancer. Cancer Chemother Pharmacol 63: 427-432, 2009.

3. Cheng WL, Wang CS, Huang YH, Tsai MM, Liang Y and Lin KH: Overexpression of CXCL1 and its receptor CXCR2 promote tumor invasion in gastric cancer. Ann Oncol 22: 2267-2276, 2011.
4. Lee HJ, Huang SM, Kim HY, et al: Evaluation of the combined expression of chemokine SDF-1 alpha and its receptor CXCR4 as a prognostic marker for gastric cancer. Exp Ther Med 2: 499-504, 2011.

5. Shen Z, Seppanen H, Vainionpaa S, et al: IL10, IL11, IL18 are differently expressed in CD14(+) TAMs and play different role in regulating the invasion of gastric cancer cells under hypoxia. Cytokine 59: 352-357, 2012.

6. Song IC, Liang ZL, Lee JC, et al: Expression of stromal cellderived factor- $1 \alpha$ is an independent risk factor for lymph node metastasis in early gastric cancer. Oncol Lett 2: 1197-1202, 2011.

7. Fiegl M, Samudio I, Clise-Dwyer K, Burks JK, Mnjoyan Z and Andreeff M: CXCR4 expression and biologic activity in acute myeloid leukemia are dependent on oxygen partial pressure. Blood 113: 1504-1512, 2009.

8. Muller A, Homey B, Soto $\mathrm{H}$, et al: Involvement of chemokine receptors in breast cancer metastasis. Nature 410: 50-56, 2001.

9. Richard CL, Tan EY and Blay J: Adenosine upregulates CXCR4 and enhances the proliferative and migratory responses of human carcinoma cells to CXCL12/SDF-1alpha. Int J Cancer 119: 2044-2053, 2006.

10. Sun X, Cheng G, Hao M, et al: CXCL12/CXCR4/CXCR7 chemokine axis and cancer progression. Cancer Metastasis Rev 29: 709-722, 2010.

11. Wang Y, Li Z, Zhang H, et al: HIF-1alpha and HIF-2alpha correlate with migration and invasion in gastric cancer. Cancer Biol Ther 10: 376-382, 2010.

12. Wang X, Li C, Chen Y, et al: Hypoxia enhances CXCR4 expression favoring microglia migration via HIF-1alpha activation. Biochem Biophys Res Commun 371: 283-288, 2008.

13. Schioppa T, Uranchimeg B, Saccani A, et al: Regulation of the chemokine receptor CXCR4 by hypoxia. J Exp Med 198: 1391-1402, 2003.

14. Krishnamachary B, Berg-Dixon S, Kelly B, et al: Regulation of colon carcinoma cell invasion by hypoxia-inducible factor 1 . Cancer Res 63: 1138-1143, 2003.

15. Ishikawa T, Nakashiro K, Klosek SK, et al: Hypoxia enhances CXCR4 expression by activating HIF-1 in oral squamous cell carcinoma. Oncol Rep 21: 707-712, 2009.

16. Cronin PA, Wang JH and Redmond HP: Hypoxia increases the metastatic ability of breast cancer cells via upregulation of CXCR4. BMC Cancer 10: 225, 2010.

17. Schutyser E, Su Y, Yu Y, et al: Hypoxia enhances CXCR4 expression in human microvascular endothelial cells and human melanoma cells. Eur Cytokine Netw 18: 59-70, 2007.

18. Lee HJ, Lee K, Lee DG, et al: Chemokine (C-X-C motif) ligand 12 is associated with gallbladder carcinoma progression and is a novel independent poor prognostic factor. Clin Cancer Res 18: 3270-3280, 2012.

19. Kwak MK, Hur K, Park DJ, et al: Expression of chemokine receptors in human gastric cancer. Tumour Biol 26: 65-70, 2005.

20. Yasumoto K, Koizumi K, Kawashima A, et al: Role of the CXCL12/CXCR4 axis in peritoneal carcinomatosis of gastric cancer. Cancer Res 66: 2181-2187, 2006.

21. Lee HJ, Kim SW, Kim HY, et al: Chemokine receptor CXCR4 expression, function, and clinical implications in gastric cancer. Int J Oncol 34: 473-480, 2009.

22. Pituch-Noworolska A, Drabik G, Szatanek R, et al: Immunophenotype of isolated tumour cells in the blood, bone marrow and lymph nodes of patients with gastric cancer. Pol J Pathol 58: 93-97, 2007.

23. Sun XJ, Sun KL, Zheng ZH, et al: Gene expression patterns in gastric cancer. Zhonghua Yi Xue Yi Chuan Xue Za Zhi 23: 142-146, 2006.

24. Hashimoto I, Koizumi K, Tatematsu M, et al: Blocking on the CXCR4/mTOR signalling pathway induces the anti-metastatic properties and autophagic cell death in peritoneal disseminated gastric cancer cells. Eur J Cancer 44: 1022-1029, 2008.

25. Tsuboi K, Kodera Y, Nakanishi H, et al: Expression of CXCL12 and CXCR4 in pT3-stage gastric cancer does not correlate with peritoneal metastasis. Oncol Rep 20: 1117-1123, 2008.

26. Iwasa S, Yanagawa T, Fan J and Katoh R: Expression of CXCR4 and its ligand SDF-1 in intestinal-type gastric cancer is associated with lymph node and liver metastasis. Anticancer Res 29: 4751-4758, 2009.

27. Arigami T, Natsugoe S, Uenosono $\mathrm{Y}$, et al: CCR7 and CXCR4 expression predicts lymph node status including micrometastasis in gastric cancer. Int J Oncol 35: 19-24, 2009. 
28. Zhao BC, Wang ZJ, Mao WZ, et al: CXCR4/SDF-1 axis is involved in lymph node metastasis of gastric carcinoma. World $\mathrm{J}$ Gastroenterol 17: 2389-2396, 2011.

29. Ding YL, Zhang JL, Tang SF, Fu QY and Li ZT: Effect of chemokine stromal cell derived factor- 1 and its receptor CXCR4 on the peritoneal carcinometastasis of gastric cancer. Zhonghua Yi Xue Za Zhi 88: 202-205, 2008 (In Chinese).

30. Koizumi K, Kato S, Sakurai H, Hashimoto I, Yasumoto K and Saiki I: Therapeutics target of CXCR4 and its downstream in peritoneal carcinomatosis of gastric cancer. Front Biosci (Schol Ed) 4: 269-276, 2012.

31. Noda S, Yashiro M, Nshii T and Hirakawa K: Hypoxia upregulates adhesion ability to peritoneum through a transforming growth factor-beta-dependent mechanism in diffuse-type gastric cancer cells. Eur J Cancer 46: 995-1005, 2010.

32. Hockel M, Schlenger K, Hockel S and Vaupel P: Hypoxic cervical cancers with low apoptotic index are highly aggressive. Cancer Res 59: 4525-4528, 1999.

33. Zhong H, De Marzo AM, Laughner E, et al: Overexpression of hypoxia-inducible factor lalpha in common human cancers and their metastases. Cancer Res 59: 5830-5835, 1999.

34. Hynes RO: Metastatic potential: generic predisposition of the primary tumor or rare, metastatic variants-or both? Cell 113 821-823, 2003

35. Griffiths EA, Pritchard SA, Welch IM, Price PM and West CM: Is the hypoxia-inducible factor pathway important in gastric cancer? Eur J Cancer 41: 2792-2805, 2005.

36. Hockel M, Schlenger K, Aral B, Mitze M, Schaffer U and Vaupel P: Association between tumor hypoxia and malignant progression in advanced cancer of the uterine cervix. Cancer Res 56: 4509-4515, 1996.

37. Generali D, Berruti A, Brizzi MP, et al: Hypoxia-inducible factor-1alpha expression predicts a poor response to primary chemoendocrine therapy and disease-free survival in primary human breast cancer. Clin Cancer Res 12: 4562-4568, 2006.

38. Bachtiary B, Schindl M, Potter R, et al: Overexpression of hypoxia-inducible factor lalpha indicates diminished response to radiotherapy and unfavorable prognosis in patients receiving radical radiotherapy for cervical cancer. Clin Cancer Res 9: 2234-2240, 2003 .

39. Schindl M, Schoppmann SF, Samonigg H, et al: Overexpression of hypoxia-inducible factor lalpha is associated with an unfavorable prognosis in lymph node-positive breast cancer. Clin Cancer Res 8: 1831-1837, 2002.
40. Pan J, Mestas J, Burdick MD, et al: Stromal derived factor-1 (SDF-1/CXCL12) and CXCR4 in renal cell carcinoma metastasis. Mol Cancer 5: 56, 2006.

41. Phillips RJ, Mestas J, Gharaee-Kermani M, et al: Epidermal growth factor and hypoxia-induced expression of CXC chemokine receptor 4 on non-small cell lung cancer cells is regulated by the phosphatidylinositol 3-kinase/PTEN/AKT/mammalian target of rapamycin signaling pathway and activation of hypoxia inducible factor-1alpha. J Biol Chem 280: 22473-22481, 2005.

42. Shim H, Lau SK, Devi S, Yoon Y, Cho HT and Liang Z: Lower expression of CXCR4 in lymph node metastases than in primary breast cancers: potential regulation by ligand-dependent degradation and HIF-1alpha. Biochem Biophys Res Commun 346: 252-258, 2006.

43. Kim SW, Kim HY, Lee HJ, Yun HJ, Kim S and Jo DY: Dexamethasone and hypoxia upregulate CXCR4 expression in myeloma cells. Leuk Lymphoma 50: 1163-1173, 2009.

44. Sun X, Wei L, Chen Q and Terek RM: CXCR4/SDF1 mediate hypoxia induced chondrosarcoma cell invasion through ERK signaling and increased MMP1 expression. Mol Cancer 9: 17, 2010.

45. Dunn LK, Mohammad KS, Fournier PG, et al: Hypoxia and TGF-beta drive breast cancer bone metastases through parallel signaling pathways in tumor cells and the bone microenvironment. PloS One 4: e6896, 2009.

46. Zagzag D, Lukyanov Y, Lan L, et al: Hypoxia-inducible factor 1 and VEGF upregulate CXCR4 in glioblastoma: implications for angiogenesis and glioma cell invasion. Lab Invest 86: 1221-1232, 2006.

47. Mahaseth $\mathrm{H}$ and Kaufman J: Optimizing stem cell collection through CXCR4 antagonists. Front Biosci (Schol Ed) 4: 611-619, 2012.

48. Gangadhar T, Nandi S and Salgia R: The role of chemokine receptor CXCR4 in lung cancer. Cancer Biol Ther 9: 409-416, 2010.

49. Iwanaga T, Iwasaki Y, Ohashi M, Nunobe $\mathrm{S}$ and Iwagami S: Establishment of a CXCR4-expressing gastric cancer cell line in nude mice and the effect of AMD 3100 on tumor regression. Gan To Sagaku Ryoho 34: 1917-1919, 2007 (In Japanese). 